\title{
Characteristics of tau oligomers
}

\section{Yan Ren and Naruhiko Sahara*}

Department of Neuroscience, Center for Translational Research in Neurodegenerative Disease, College of Medicine, University of Florida, Gainesville, FL, USA

\section{Edited by:}

Jesus Avila, Centro de Biología Molecular Severo Ochoa CSIC-UAM, Spain

\section{Reviewed by:}

Jesus Avila, Centro de Biología Molecular Severo Ochoa CSIC-UAM, Spain

Emmanuel Planel, Centre Hospitalier de l'université Laval, Canada

*Correspondence:

Naruhiko Sahara, Department of Neuroscience, Center for Translational Research in Neurodegenerative

Disease, College of Medicine, University of Florida, 1275 Center

Drive, Gainesville, FL, 32610, USA

e-mail: nsahara@ufl.edu
In Alzheimer disease (AD) and other tauopathies, microtubule-associated protein tau becomes hyperphosphorylated, undergoes conformational changes, aggregates, eventually becoming neurofibrillary tangles (NFTs). As accumulating evidence suggests that NFTs themselves may not be toxic, attention is now turning toward the role of intermediate tau oligomers in AD pathophysiology. Sarkosyl extraction is a standard protocol for investigating insoluble tau aggregates in brains. There is a growing consensus that sarkosyl-insoluble tau correlates with the pathological features of tauopathy. While sarkosyl-insoluble tau from tauopathy brains has been well characterized as a pool of filamentous tau, other dimers, multimers, and granules of tau are much less well understood. There are protocols for identifying these tau oligomers. In this mini review, we discuss the characteristics of tau oligomers isolated via different methods and materials.

Keywords: tau, oligomers, dimer, sarkosyl-insoluble, antibody

\section{INTRODUCTION}

Tau is a phospho-protein that belongs to the family of microtubule (MT)-associated proteins. The primary function of tau protein is to modulate MT dynamics for maintaining neuronal processes and regulating axonal transport. During pathogenesis, tau protein abnormally aggregates into intracellular, filamentous inclusions, or neurofibrillary tangles (NFTs) in the brains of individuals with neurodegenerative disorders. These are termed tauopathies [reviewed in Ref. (1)].

In human tauopathies, intracellular aggregates of abnormally hyperphosphorylated tau protein and neuronal cell loss typically coincide within the same brain regions (2). Several transgenic mouse models that overexpress human tau protein have demonstrated how tau pathology and neuronal loss progresses [mouse models are reviewed in Ref. (3)]. However, recent data suggest that tau is involved in neuronal dysfunction before NFTs are formed $(4,5)$. In vitro tau polymerization studies indicated that NFT formation consists of several steps: dimerization, multimerization, oligomerization, and protofibril formation (6-11).

About two decades ago, tau aggregation intermediates (also referred to as $\mathrm{AD}$ P-tau) were isolated from the buffer-soluble fraction derived from brains of $\mathrm{AD}$ patients (12). More than 10 years later, attention has focused on oligomeric tau species in human (13) and transgenic mouse (14) brains in order to identify the exact neurotoxic components of tau protein. However, the potential role of tau oligomers is poorly understood because they exist in various states (e.g., dimers, multimers, and granules). Here, we review various protocols used to isolate tau oligomers and propose a general outline for the identification of tau oligomers.

\section{SOLUBLE PRE-FIBRILLAR TAU IN HUMAN AD BRAINS}

Greenberg and Davies first reported to isolate sarkosyl-insoluble tau from paired helical filament (PHF)-enriched fraction from human $\mathrm{AD}$ brain homogenates (15). Cortical gray matter was homogenized in buffer containing $10 \mathrm{mM}$ Tris- $\mathrm{HCl}(\mathrm{pH} \mathrm{7.4)}$, $1 \mathrm{mM}$ EGTA, $0.8 \mathrm{M} \mathrm{NaCl}$, and $10 \%$ sucrose, and then centrifuged at $27,200 \times g$. PHF-associated tau was enriched from the supernatant by taking advantage of their insolubility in the presence of a detergent, sarkosyl. PHF-associated tau migrated at around $57-68 \mathrm{kDa}$ on one-dimensional PAGE gels. This enriched supernatant was more acidic on two-dimensional PAGE gels compared to extracts from normal brains. Although this PHF-associated tau was not extracted from highly insoluble fraction containing NFTs, the sarkosyl-insoluble tau displayed the same structural and antigenic properties as PHFs isolated from NFTs (16-18) and was distinguishable from normal, soluble tau proteins.

Kopke et al. isolated non-PHF hyperphosphorylated tau from $\mathrm{AD}$ brains (12). In their protocol, cortical gray matter was homogenized in buffer containing $20 \mathrm{mM}$ Tris- $\mathrm{HCl}(\mathrm{pH} 8.0), 0.32 \mathrm{M}$ sucrose, $10 \mathrm{mM} \beta$-mercaptoethanol, $5 \mathrm{mM}$ EGTA, $1 \mathrm{mM}$ EDTA, $5 \mathrm{mM} \mathrm{MgSO}_{4}$, and proteinase inhibitors. The homogenate was then subjected to differential centrifugation, and the fraction resulting from centrifugation between 27,000 and $200,000 \times g$ was collected. This fraction was further extracted with $8 \mathrm{M}$ urea to separate out the PHF-enriched pool. The supernatant contained abnormally phosphorylated non-PHF tau. These tau species were named AD P-tau and had a molecular weight of $67-70 \mathrm{kDa}$. They were three to fourfold more phosphorylated than tau extracted from control brains and could be detected by Taul antibody after alkaline phosphatase treatment. These highly phosphorylated AD P-tau proteins lost their normal MT assembly-promoting activity, which could be recovered upon dephosphorylation with alkaline phosphatase (19). Moreover, AD P-tau could sequester normal tau into filamentous tau aggregates, resulting in MT de-polymerization (20).

These studies suggest that a pool of intermediate pathological tau species exists and that this pool can be recovered in 
buffer-soluble fractions. The physiological activity and function of these tau species is reduced compared to normal tau species due to hyperphosphorylation. Since the intracellular mobility dynamics of these intermediate tau species is much greater than that of condensed tau aggregates in NFTs, it is possible that intermediate tau species induce neuronal death and/or synaptic dysfunction. Therefore, the isolation and characterization of these tau species is paramount for understanding the pathogenesis of $\mathrm{AD}$ and for searching therapeutic methods.

\section{TAU OLIGOMERS IN MOUSE MODELS OF TAUOPATHY}

The first tau transgenic mouse model of frontotemporal dementia with Parkinsonism linked to tau on chromosome 17 (FTDP-17Tau) was the JNPL3 line, which overexpresses P301L mutant $4 \mathrm{R} 0 \mathrm{~N}$ tau (21). The biochemical characterization of insoluble tau in these mice was done by a modified Greenberg and Davies method $(21,22)$. In this protocol, mouse brains were homogenized in buffer containing $25 \mathrm{mM}$ Tris- $\mathrm{HCl}$ ( $\mathrm{pH} 7.4$ ), $150 \mathrm{mM} \mathrm{NaCl}, 1 \mathrm{mM}$ EDTA, $1 \mathrm{mM}$ EGTA, phosphatase inhibitors, and protease inhibitors. A pellet collected from $150,000 \times g$ centrifugation was re-homogenized in high-salt/sucrose buffer [10 mM Tris- $\mathrm{HCl}$ ( $\mathrm{pH} 7.4), 0.8 \mathrm{M} \mathrm{NaCl}, 10 \%$ sucrose, $1 \mathrm{mM}$ EGTA, $1 \mathrm{mM}$ PMSF] and centrifuged again at $150,000 \times g$. The resulting supernatant was incubated with $1 \%$ sarkosyl, and then centrifuged at $150,000 \times g$. The pellet was resuspended in TBS as the sarkosyl-insoluble fraction. A $64-\mathrm{kDa}$ tau predominantly existed in the sarkosyl-insoluble fraction; this tau was phosphorylated at multiple sites. Most notably, the amount of $64 \mathrm{kDa}$ tau increased in an age-dependent manner, correlating well with the pathogenesis in JNPL3 mouse brain.

Noble et al. proposed a slightly different protocol for tissue homogenization using RIPA buffer without SDS (23). This modification allows for the study of both cytosolic and membraneassociated proteins involved in $\mathrm{AD}$ pathogenesis, such as amyloid precursor protein (APP), in the same extracts (24).

Another broadly used tauopathy mouse model is rTg4510 mice, which express repressible P301L mutant 4R0N tau and develop progressive age-related NFTs, neuronal loss, and behavioral impairment (5). Using a protocol similar to the one for JNPL3 mice with an additional $13,000 \times g$ centrifugation as the first step, Berger et al. identified 140 and $170 \mathrm{kDa}$ multimeric tau species in rTg4510 mouse brains (14). The $140 \mathrm{kDa}$ tau was recovered in the supernatant fraction resulting from $150,000 \times g$ centrifugation, while the $170 \mathrm{kDa}$ tau was mostly in the sarkosyl-insoluble fraction. Both multimers were not affected by the presence or absence of reducing agent, indicating that the multimers are disulfidebond independent. Importantly, the accumulation of $140 \mathrm{kDa}$ tau coincided with the behavioral impairments of rTg4510 mice (14). Although this finding has had a huge impact on our understanding the neurotoxic mechanisms of tau oligomers, it is still unclear whether 140 and $170 \mathrm{kDa}$ tau multimers can induce neuronal dysfunction. This is because these multimers comprise such a small proportion of the total tau pool (roughly $<0.1 \%$ of total tau, as estimated by Western blot signal). It should be noted that tau multimers with apparent molecular weights of $\sim 140$ and $\sim 170 \mathrm{kDa}$ are in fact tau dimers of $\sim 120$ and $\sim 130 \mathrm{kDa}$, based on Bis-Tris or Tris-acetate SDS-PAGE migration $(11,25)$. This was further supported by mass spectrometry analysis of cross-linked tau dimers (26).

Most recently, we demonstrated that TBS-extractable $64 \mathrm{kDa}$ tau species represents better the species involved in the progression of brain atrophy than does the sarkosyl-insoluble tau species (25). These $64 \mathrm{kDa}$ tau species can be recovered in the supernatant following centrifugation of brain homogenates at $27,000 \times g$ and further separation from normal tau by $150,000 \times g$ centrifugation. TBS-extractable $64 \mathrm{kDa}$ tau and normal tau are similar in thermo-stability but differ in other properties. Under non-reducing gel electrophoresis conditions, nearly all $64 \mathrm{kDa}$ tau species are detected as dimers $(\sim 130 \mathrm{kDa}$, according to size of molecular markers), whereas most normal tau proteins are detected as monomers. Immuno-electron microscopy revealed that the TBS-extractable $64 \mathrm{kDa}$ tau enriched fraction contains tau-positive granules and filaments (25). This morphological finding was supported by MC1 immunoreactivity and Ab39 insensitivity (25). The MC1 antibody recognizes an early pathogenic conformation of tau (27), while the Ab39 antibody only detects mature tangles $(28,29)$. Overall, the characteristics of TBS-extractable $64 \mathrm{kDa}$ tau are similar to AD P-tau from human brains.

\section{IN VITRO TAU OLIGOMERIZATION}

With tau assembly modeled in vitro, unphosphorylated recombinant tau can be polymerized by inducers such as heparin, heparan sulfate, polyunsaturated fatty acids, RNA, or quinones (30-34). Using the heparin-induced tau self-assembly method, we produced and isolated granular-shaped tau oligomers from soluble tau and filamentous tau by sucrose density gradient ultracentrifugation (10). These granular tau oligomers were morphologically defined by atomic force microscopy (AFM) to be $15-25 \mathrm{~nm}$ granules, and their molecular mass corresponded to about 40 tau molecules (10). Once formed, granular tau can continue to form filaments without any inducers in a concentration-dependent manner (10).

More recently, Lasagna-Reeves et al. proposed a method to prepare tau oligomers by using amyloid seeds (35). In their protocol, tau oligomerization can be induced in a relatively short period ( $1 \mathrm{~h}$ incubation at room temperature) after adding A $\beta 42$ oligomers. After a total of three rounds of seeding procedures, $A \beta 42$ seeds could be diluted to below the detection limit (35). Examination of these tau oligomers by transmission electron microscopy or AFM revealed a spherical morphology (35). This unique method of producing tau oligomers is a reasonable representative model supporting the amyloid hypothesis $(36,37)$, in which $\mathrm{A} \beta$ oligomers trigger NFT formation. Interestingly, tau oligomers, but not tau monomers or tau fibrils, can cause memory impairment in wild-type mice (13) and can decrease long-term potentiation in hippocampal brain slices (38).

The production of granular tau oligomers must be initiated by dimerization of tau monomers. Heparin-induced tau polymerization allows us to detect initial dimers because of the 
relatively slower kinetics compared to arachidonic acid-induced tau polymerization $(6,39)$. It begins by increasing the formation of cysteine-dependent dimers, which occur prior to the detection of thioflavin $\mathrm{T}$ (ThT) binding (11). The kinetics of tau polymerization is dependent on oxidative/reducing state. Higherorder oligomers and aggregates assemble more rapidly in the absence of the reducing agent dithiothreitol (DTT) (11). However, cysless-tau (4R tau with both C291A and C322A mutations) forms dimers, which eventually aggregate into fibrils after $24 \mathrm{~h}$ incubation with heparin, suggesting that tau aggregation occurs without disulfide-bond formation (11).

Two distinct tau dimers (i.e., cysteine-dependent and cysteineindependent dimers) have been identified in tauopathy mouse models, including JNPL3 mice (11) and $\operatorname{rTg} 4510$ mice $(14,25)$. These dimers have also been shown in cell cultures $(11,40)$. Dimer formation is an essential step for their further assembly into higher-order oligomers. Although these dimers themselves may not exist in a steady state, it is important to detect the initial step of tau dimerization.

\section{GENERATION OF TAU OLIGOMER-SPECIFIC ANTIBODIES}

A monoclonal antibody that selectively recognizes tau dimers and higher-order oligomers has been generated by Binder's group (26). This antibody, named tau oligomeric complex 1 (TOC1), was made against benzophenone-4-maleimide cross-linked recombinant tau dimers (26). Immunogold labeling and dot-blot analysis of aggregated recombinant tau revealed that TOC1 selectively labels tau dimers or oligomers but not filaments (26). Based on their preliminary mapping of the TOC1 epitope, the proline-rich region (Gly155-Gln244) and the Cterminal portion (Leu376-Ser421) were identified as potential binding segments for forming cross-linked tau dimer (26). Since these two regions are on the opposite sides of the MT-binding domains, Patterson et al. advanced the idea of the formation of an antiparallel dimer (26).

TOC1 immunoreactivity is selectively detectable in the early stage of $\mathrm{AD}$ pathogenesis; however, TOC1 antibody fails to label mature tangles in $\mathrm{AD}$ brains (26). In rTg4510 mice, TOC1 immunoreactivity was observed in the TBS-extractable $64 \mathrm{kDa}$ tau enriched fraction and linked to early pathological changes (Sahara, in preparation). Notably, the immunohistochemical staining pattern of TOC1 antibody was clearly different from those of $\mathrm{MC1}$ and $\mathrm{Ab39}$ antibodies (Figure 1). Since NFTs themselves might be protective [reviewed in Ref. (41)], other harmful tau species such as tau oligomers are currently of particular interest. If TOC1 antibody selectively interacts with tau dimers and higher-order oligomers but not tau filaments, and if those species cause neurotoxicity, this antibody can be a useful tool to track the pathway of tau neurotoxicity.

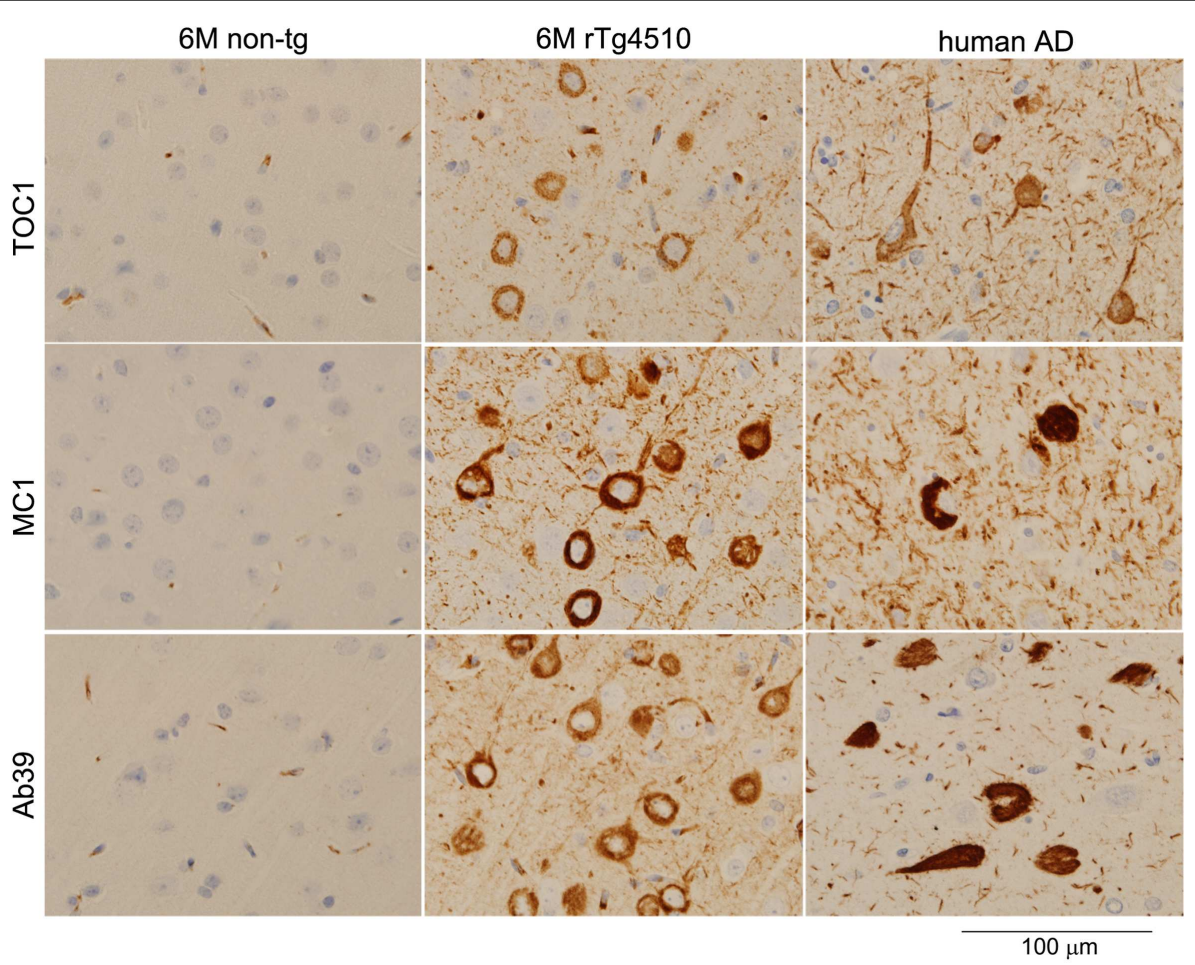

FIGURE 1 | Light microscopic images of immunostained brain sections from a non-transgenic mouse, a rTg4510 mouse, and human with $A D$. Formalin-fixed paraffin sections were stained with TOC1 (1:2500), MC1 (1:1000), and Ab39 (1:250) antibodies by the Dako Universal Autostainer (Dako, Carpinteria, CA, USA). The sections were then counterstained with hematoxylin. TOC1 diffusely stained cytoplasmic regions of neurons (top panels, rTg4510 and AD brains), while MC1 and Ab39 densely stained these neurons (middle and bottom panels, respectively). This difference in staining pattern is due to the specific binding of TOC1 antibody to premature tau aggregates. Scale Bar, $100 \mu \mathrm{m}$. 
Table 1 | Summary of brain-derived tau oligomer preparation methods.

\begin{tabular}{|c|c|c|c|c|}
\hline Product & Reference & Material origin & Detect method & Oligomer properties \\
\hline AD P-tau & $\begin{array}{l}\text { Kopke et al. (12), Alonso } \\
\text { et al. (20), Alonso et al. } \\
\text { (44) }\end{array}$ & AD patients & WB, EM & $\begin{array}{l}\text { AD P-tau is isolated from the } 27 \mathrm{~K}-200 \mathrm{~K} \times g \text { fraction, } \\
\text { soluble in urea, hyperphosphorylated, no ubiquitin } \\
\text { immunoreactivity, self-assembly into filaments, } \\
\text { sequesters N-tau }\end{array}$ \\
\hline $\begin{array}{l}140 \text { and } 170 \mathrm{kDa} \\
\text { tau }\end{array}$ & Berger et al. (14) & $\begin{array}{l}\text { rTg4510 mice } \\
\text { JNPL3 mice }\end{array}$ & WB, SEC & $\begin{array}{l}\text { Disulfide-bond independent, correlates with memory } \\
\text { loss, } 140 \mathrm{kDa} \text { tau is not hyperphosphorylated, } 170 \mathrm{kDa} \\
\text { tau is hyperphosphorylated, and has strong } \\
\text { immunoreactivity with AT8 }\end{array}$ \\
\hline $\begin{array}{l}\text { TBS-extractable } \\
64 \text { kDa tau }\end{array}$ & Sahara et al. (25) & rTg4510 mice & WB, IHC, EM & $\begin{array}{l}\text { TBS-extractable } 64 \mathrm{kDa} \text { tau is isolated from } 27 \mathrm{~K} \text { to } \\
150 \mathrm{~K} \times g \text { fraction, thermo-stable, hyperphosphorylated, } \\
\text { mostly disulfide-bond-dependent, correlates with brain } \\
\text { atrophy, contains tau-positive granules/short filaments }\end{array}$ \\
\hline T22-positive tau & $\begin{array}{l}\text { Lasagna-Reeves et al. } \\
\text { (38), Lasagna-Reeves et } \\
\text { al. (42) }\end{array}$ & AD patients & $\begin{array}{l}\text { WB, IHC, SEC, } \\
\text { AFM }\end{array}$ & $\begin{array}{l}\text { Hyperphosphorylated, not ubiquitinated at pretangle } \\
\text { stage, contains oligomers with } 4-8 \mathrm{~nm} \text { diameters, } \\
\text { propagate abnormal tau conformation of endogenous } \\
\text { murine tau }\end{array}$ \\
\hline
\end{tabular}

WB, Western blot; EM, electronic microscopy; IHC, immunohistochemistry; SEC, size-exclusion chromatography; AFM, atomic force microscopy.

Another tau oligomer-specific antibody, T22, was generated by Kayed's group (42) against antigenic tau prepared from $\mathrm{A} \beta$ seeding of tau oligomers (35). The specificity of this antibody was confirmed by ELISA and dot blotting. It detects only tau oligomers but not tau monomers or PHF fibrils prepared by the heparin-induced tau polymerization method (42). Immunohistochemically, this antibody selectively stains pretangles, neuritic plaques, and neuropil threads but not ghost tangles in $\mathrm{AD}$ brain sections (42). Western blotting showed that T22 antibody recognizes higher-molecular-weight tau species (e.g., dimers, trimers, and tetramers) but not monomers (42). It should be noted that the SDS-PAGE samples were not denatured by boiling before running on gels and that T22 immunoreactivity was diminished by denaturing agents such as $8 \mathrm{M}$ urea (42).

These novel tau oligomer-specific antibodies provide a new method to diagnose the early pathological changes that occur in tauopathy. It would be extremely useful to develop methods employing cerebrospinal fluid biomarkers combined with total tau, phosphorylated tau, tau oligomers, and other biomarker measurements to differentially diagnose dementias, such as $\mathrm{AD}$, frontotemporal lobar degeneration, progressive supranuclear palsy, corticobasal degeneration, dementia with Lewy bodies, vascular dementia, and prion disease.

\section{CONCLUSION}

Abnormal tau aggregation is considered to be a critical pathological feature of tauopathy. However, the initial molecular event of tau pathogenesis is yet unclear. The hyperphosphorylation of tau is strongly suggested to be directly correlated with the severity of AD pathology (43). Iqbal and colleagues demonstrated that hyperphosphorylated tau extracted from $\mathrm{AD}$ brain reduces
MT stabilization, sequesters normal tau from MT, and aggregates themselves in the absence of inducer molecules (20, 44). Many studies attempting to identify tau oligomers have demonstrated the existence of hyperphosphorylated tau oligomers in human and transgenic mouse brains (e.g., AD P-tau, 140 and $170 \mathrm{kDa}$ tau multimers, TBS-extractable $64 \mathrm{kDa}$ tau, and T22 antibodypositive tau oligomers) (Table 1). Thus, hyperphosphorylation of tau could be the initial event of NFT formation. However, the amount of $140 \mathrm{kDa}$ tau multimer (normal tau dimer) correlates well with behavioral deficits (14), suggesting that hyperphosphorylated tau oligomers may not be essential for neurotoxicity. If antibodies can be generated to recognize non-phosphorylated and hyperphosphorylated tau dimers independently, we will be able to better identify toxic tau species and optimize potential oligomerization inhibitors as possible novel therapies. Standardized isolation methods of tau oligomers are in need to improve consistency between researchers.

In summary, accumulating evidence from biochemistry, immunology, and molecular imaging reveal the existence of tau oligomers as mainly buffer-soluble, non-filamentous, granularshaped conformers. The neurotoxicity of these oligomers has been confirmed in both in vitro and in vivo experiments $(4,5,14,42,45)$. The next step of tau oligomer research should investigate whether tau dimers and/or non-granular oligomers exist and functionally correspond to neuronal dysfunction.

\section{ACKNOWLEDGMENTS}

Work in the authors' laboratory is supported in part by an R21 grant (NS067127) from the National Institutes of Health/National Institute of Neurological Diseases and Stroke, and a grant from the Thomas H. Maren Junior Investigator Fund of the University of Florida. 


\section{REFERENCES}

1. Lee VM, Goedert M, Trojanowski JQ. Neurodegenerative tauopathies. Annu Rev Neurosci (2001) 24:112159. doi:10.1146/annurev.neuro.24. 1.1121

2. Gomez-Isla T, Hollister R, West $\mathrm{H}, \quad$ Mui S, Growdon $\mathrm{JH}$, Petersen RC, et al. Neuronal loss correlates with but exceeds neurofibrillary tangles in Alzheimer's disease. Ann Neurol (1997) 41:17-24. doi: 10.1002/ana.410410106

3. Denk F, Wade-Martins R. Knockout and transgenic mouse models of tauopathies. Neurobiol Aging (2009) 30:1-13. doi:10.1016/j. neurobiolaging.2007.05.010

4. Wittmann CW, Wszolek MF, Shulman JM, Salvaterra PM, Lewis J, Hutton M, et al. Tauopathy in Drosophila: neurodegeneration without neurofibrillary tangles. Science (2001) 293:711-4. doi: $10.1126 /$ science. 1062382

5. Santacruz K, Lewis J, Spires T, Paulson J, Kotilinek L, Ingelsson $\mathrm{M}$, et al. Tau suppression in a neurodegenerative mouse model improves memory function. Science (2005) 309:476-81. doi:10. 1126/science.1113694

6. Barghorn S, Mandelkow E. Toward a unified scheme for the aggregation of tau into Alzheimer paired helical filaments. Biochemistry (2002) 41:14885-96. doi:10. 1021/bi026469j

7. Makrides V, Shen TE, Bhatia R, Smith BL, Thimm J, Lal R, et al. Microtubule-dependent oligomerization of tau. Implications for physiological tau function and tauopathies. J Biol Chem (2003) 278:33298-304. doi: 10.1074/jbc.M305207200

8. Chirita CN, Kuret J. Evidence for an intermediate in tau filament formation. Biochemistry (2004) 43:1704-14. doi:10.1021/bi036034b

9. Kuret J, Chirita CN, Congdon EE, Kannanayakal T, Li G, Necula M, et al. Pathways of tau fibrillization. Biochim Biophys Acta (2005) 1739:167-78. doi:10.1016/j.bbadis. 2004.06.016

10. Maeda S, Sahara N, Saito Y, Murayama M, Yoshiike Y, Kim H, et al. Granular tau oligomers as intermediates of tau filaments. Biochemistry (2007) 46:3856-61. doi: 10.1021/bi061359o

11. Sahara N, Maeda S, Murayama M, Suzuki T, Dohmae N, Yen S-H, et al. Assembly of two distinct dimers and higher-order oligomers from full-length tau. Eur J Neurosci
(2007) 25:3020-9. doi:10.1111/j. 1460-9568.2007.05555.x

12. Kopke E, Tung YC, Shaikh S, Alonso AC, Iqbal K, Grundke-Iqbal I. Microtubule-associated protein tau. Abnormal phosphorylation of a non-paired helical filament pool in Alzheimer disease. J Biol Chem (1993) 268:24374-84.

13. Lasagna-Reeves CA, CastilloCarranza DL, Sengupta U, Clos AL, Jackson GR, Kayed R. Tau oligomers impair memory and induce synaptic and mitochondrial dysfunction in wild-type mice. Mol Neurodegener (2011) 6:39. doi: 10.1186/1750-1326-6-39

14. Berger Z, Roder H, Hanna A, Carlson A, Rangachari V, Yue $M$, et al. Accumulation of pathological tau species and memory loss in a conditional model of tauopathy. $J$ Neurosci (2007) 27:3650-62. doi:10. 1523/JNEUROSCI.0587-07.2007

15. Greenberg SG, Davies P. A preparation of Alzheimer paired helical filaments that displays distinct tau proteins by polyacrylamide gel electrophoresis. Proc Natl Acad Sci U S A (1990) 87:5827-31. doi:10.1073/ pnas.87.15.5827

16. Selkoe DJ. Altered structural proteins in plaques and tangles: what do they tell us about the biology of Alzheimer's disease? Neurobiol Aging (1986) 7:425-32. doi: 10.1016/0197-4580(86)90055-2

17. Wisniewski HM, Iqbal K, GrundkeIqbal I, Rubenstein R. The solubility controversy of paired helical filaments: a commentary. Neurochem Res (1987) 12:93-5. doi:10.1007/ BF00971370

18. Ksiezak-Reding H, Binder LI, Yen SH. Alzheimer disease proteins (A68) share epitopes with tau but show distinct biochemical properties. J Neurosci Res (1990) 25:420 30. doi:10.1002/jnr.490250320

19. Alonso AC, Zaidi T, Grundke-Iqbal I, Iqbal K. Role of abnormally phosphorylated tau in the breakdown of microtubules in Alzheimer disease. Proc Natl Acad Sci U S A (1994) 91:5562-6. doi:10.1073/pnas.91.12. 5562

20. Alonso AC, Grundke-Iqbal I, Iqbal K. Alzheimer's disease hyperphosphorylated tau sequesters normal tau into tangles of filaments and disassembles microtubules. Nat Med (1996) 2:783-7. doi:10.1038/ nm0796-783

21. Lewis J, Mcgowan E, Rockwood J, Melrose H, Nacharaju P, Van Slegtenhorst M, et al. Neurofibrillary tangles, amyotrophy and progressive motor disturbance in mice expressing mutant (P301L) tau protein. Nat Genet (2000) 25:402-5. doi:10.1038/78078

22. Sahara N, Lewis J, Deture $M$, Mcgowan E, Dickson DW, Hutton $\mathrm{M}$, et al. Assembly of tau in transgenic animals expressing P301L tau: alteration of phosphorylation and solubility. J Neurochem (2002) 83:1498-508. doi:10.1046/j. 1471-4159.2002.01241.x

23. Noble W, Planel E, Zehr C, Olm V, Meyerson J, Suleman F, et al. Inhibition of glycogen synthase kinase- 3 by lithium correlates with reduced tauopathy and degeneration in vivo. Proc Natl Acad Sci U S A (2005) 102:6990-5. doi:10.1073/ pnas.0500466102

24. Julien C, Bretteville A, Planel E. Biochemical isolation of insoluble tau in transgenic mouse models of tauopathies. Methods Mo Biol (2012) 849:473-91. doi:10. 1007/978-1-61779-551-0_32

25. Sahara N, Deture M, Ren Y, Ebrahim A-S, Kang D, Knight J, et al. Characteristics of TBSextractable hyperphosphorylated Tau species: aggregation intermediates in rTg4510 mouse brain. $J$ Alzheimers Dis (2013) 33:249-63. doi:10.3233/JAD-2012-121093

26. Patterson KR, Remmers C, Fu Y, Brooker S, Kanaan NM, Vana L, et al. Characterization of prefibrillar Tau oligomers in vitro and in Alzheimer disease. J Biol Chem (2011) 286:23063-76. doi:10.1074/ jbc.M111.237974

27. Jicha GA, Bowser R, Kazam IG, Davies P. Alz-50 and MC1 , a new monoclonal antibody raised to paired helical filaments, recognize conformational epitopes on recombinant tau. J Neurosci Res (1997) 48:128$32 . \quad$ doi:10.1002/(SICI)10974547(19970415)48:2<128::AIDJNR5 > 3.0.CO;2-E

28. Yen SH, Dickson DW, Crowe A, Butler M, Shelanski ML. Alzheimer's neurofibrillary tangles contain unique epitopes and epitopes in common with the heat-stable microtubule associated proteins tau and MAP2. Am J Pathol (1987) 126:81-91.

29. Dickson DW, Ksiezak-Reding $H$, Liu WK, Davies P, Crowe A, Yen SH. Immunocytochemistry of neurofibrillary tangles with antibodies to subregions of tau protein: identification of hidden and cleaved tau epitopes and a new phosphorylation site. Acta Neuropathol (1992) 84:596-605. doi:10. 1007/BF00227736
30. Goedert M, Jakes R, Spillantini MG, Hasegawa M, Smith MJ, Crowther RA. Assembly of microtubule-associated protein tau into Alzheimer-like filaments induced by sulphated glycosaminoglycans. Nature (1996) 383:550-3. doi: $10.1038 / 383550 \mathrm{a} 0$

31. Kampers T, Friedhoff P, Biernat J, Mandelkow EM, Mandelkow E. RNA stimulates aggregation of microtubule-associated protein tau into Alzheimer-like paired helical filaments. FEBS Lett (1996) 399:344-9. doi: 10.1016/S0014-5793(96)01386-5

32. Perez M, Valpuesta JM, Medina M, Montejo De Garcini E, Avila J. Polymerization of tau into filaments in the presence of heparin: the minimal sequence required for tau-tau interaction. J Neurochem (1996) 67:1183-90. doi:10.1046/j. 1471-4159.1996.67031183.x

33. Wilson DM, Binder LI. Free fatty acids stimulate the polymerization of tau and amyloid beta peptides. In vitro evidence for a common effector of pathogenesis in Alzheimer's disease. Am J Pathol (1997) 150:2181-95.

34. Santa-Maria I, Hernandez F, Martin CP, Avila J, Moreno FJ. Quinones facilitate the selfassembly of the phosphorylated tubulin binding region of tau into fibrillar polymers. Biochemistry (2004) 43:2888-97. doi: 10.1021/bi035345j

35. Lasagna-Reeves CA, CastilloCarranza DL, Guerrero-Muoz MJ, Jackson GR, Kayed R. Preparation and characterization of neurotoxic tau oligomers. Biochemistry (2010) 49:10039-41. doi: 10.1021/bi1016233

36. Selkoe DJ. The molecular pathology of Alzheimer's disease. Neuron (1991) 6:487-98. doi:10.1016/08966273(91)90052-2

37. Selkoe DJ. Soluble oligomers of the amyloid beta-protein impair synaptic plasticity and behavior. Behav Brain Res (2008) 192:106-13. doi: 10.1016/j.bbr.2008.02.016

38. Lasagna-Reeves CA, CastilloCarranza DL, Sengupta U, Guerrero-Munoz MJ, Kiritoshi T, Neugebauer V, et al. Alzheimer brain-derived tau oligomers propagate pathology from endogenous tau. Sci Rep (2012) 2:700. doi: 10.1038/srep00700

39. Friedhoff P, Schneider A, Mandelkow EM, Mandelkow E. Rapid assembly of Alzheimer-like paired helical filaments from microtubuleassociated protein tau monitored by 
fluorescence in solution. Biochemistry (1998) 37:10223-30. doi:10. 1021/bi980537d

40. Zhang YJ, Xu YF, Chen XQ, Wang XC, Wang JZ. Nitration and oligomerization of tau induced by peroxynitrite inhibit its microtubule-binding activity. FEBS Lett (2005) 579:2421-7. doi: 10.1016/j.febslet.2005.03.041

41. Lee HG, Perry G, Moreira PI, Garrett MR, Liu Q, Zhu X, et al. Tau phosphorylation in Alzheimer's disease: pathogen or protector? Trends Mol Med (2005) 11:164-9. doi:10. 1016/j.molmed.2005.02.008

42. Lasagna-Reeves CA, CastilloCarranza DL, Sengupta U, Sarmiento J, Troncoso J, Jackson GR, et al. Identification of oligomers at early stages of tau aggregation in Alzheimer's disease. FASEB J (2012) 6:1946-59. doi: 10.1096/fj.11-199851

43. Augustinack JC, Schneider A, Mandelkow EM, Hyman BT. Specific tau phosphorylation sites correlate with severity of neuronal cytopathology in Alzheimer's disease. Acta Neuropathol (2002) 103:26-35. doi: 10.1007/s004010100423

44. Alonso A, Zaidi T, Novak M, Grundke-Iqbal I, Iqbal K. Hyperphosphorylation induces self-assembly of tau into tangles of paired helical filaments/straight filaments. Proc Natl Acad Sci
U S A (2001) 98:6923-8. doi: 10.1073/pnas. 121119298

45. Khlistunova I, Biernat J, Wang Y, Pickhardt M, Von Bergen M, Gazova $Z$, et al. Inducible expression of Tau repeat domain in cell models of tauopathy: aggregation is toxic to cells but can be reversed by inhibitor drugs. $J$ Biol Chem (2006) 281:1205-14. doi:10.1074/ jbc.M507753200

Conflict of Interest Statement: The authors declare that the research was conducted in the absence of any commercial or financial relationships that could be construed as a potential conflict of interest.
Received: 21 May 2013; paper pending published: 03 July 2013; accepted: 09 July 2013; published online: 19 July 2013.

Citation: Ren $Y$ and Sahara $N$ (2013) Characteristics of tau oligomers. Front. Neurol. 4:102. doi: 10.3389/fneur.2013.00102

This article was submitted to Frontiers in Neurodegeneration, a specialty of Frontiers in Neurology.

Copyright () 2013 Ren and Sahara. This is an open-access article distributed under the terms of the Creative Commons Attri-

bution License, which permits use, distribution and reproduction in other forums, provided the original authors and source are credited and subject to any copyright notices concerning any third-party graphics etc. 